\title{
Der chinesische Held - ein Opernstoff aus dem alten China im aufgeklärten Europa
}

\author{
Achim Aurnhammer
}

Am 13. Mai 1752 wurde im Gartentheater von Schloss Schönbrunn bei Wien die Oper L'eroe cinese, Der chinesische Held, uraufgeführt. Das italienische Libretto stammt von dem kaiserlichen Hofdichter Pietro Metastasio, die Musik von Giuseppe Bonno. Anlass der Aufführung war der 35. Geburtstag der Kaiserin Maria Theresia, die Aufführenden waren Hof-„Damen und Cavaliere“, darunter die Erzherzoginnen. ${ }^{1}$

Metastasios Libretto war im 18. Jahrhundert überaus populär und wurde immer wieder in Musik gesetzt, unter anderem von den namhaften Komponisten Baldassare Galuppi, Johann Adolph Hasse und Domenico Cimarosa. Eine Aufstellung über die diversen Aufführungen von Metastasios Chinesischem Helden in wechselnden Vertonungen bezeugt den enormen und europaweiten Erfolg dieses Stücks. ${ }^{2}$ Erstaunlicherweise blieb Metastasios Drama forscherlich lange unbeachtet; erst in jüngerer Zeit wurde es im Zuge der Kulturtransferforschung als bedeutendes Zeugnis der China-Mode des 18. Jahrhunderts neu gewürdigt, deren projektive Überformung des historischen China zu einem europäischen Wunsch- oder Imaginationsraum Adrian Hsia als ,Chinesia‘ bestimmt hat. Die zentrale Helden-Thematik,

1 So die Angabe (,rappresentato nell'Imperial Corte da Dame, e Cavalieri“) auf dem Titelblatt der editio princeps: Pietro Metastasio: L'eroe cinese. Dramma per musica, Palermo 1752; das Libretto, das häufig aufgelegt wurde (vgl. etwa ders.: Tutte le opere, hg. von Bruno Brunelli, Bd. 1, Turin 21965, S. 1153-1194), wird im Folgenden nach der Erstausgabe zitiert; die erste deutsche Übersetzung erschien als Bilingue anlässlich einer Aufführung in Hamburg im Juli 1754 (ders.: L'eroe cinese. Dramma per musica / Der Sinesische Held, ein musicalisches Schauspiel, Hamburg 1754). Bereits für den Karneval des Jahres 1735 hatte Metastasio mit den ,Chinesinnen' ein chinesisches Sujet für eine Festa teatrale ausgewählt: Vgl. Pietro Metastasio: Le cinesi, in: ders.: Tutte le opere, hg. von Bruno Brunelli, Bd. 2, Turin ${ }^{2} 1965$, S. 341-354. Das Stück ist durch Glucks Vertonung operngeschichtlich bedeutsam; vgl dazu Silke Leopold: Glucks „Chinesinnen“, in: Winfried Kirsch / Sieghart Döhring (Hg.): Geschichte und Dramaturgie des Operneinakters, Laaber 1991, S. 75-84.

2 Einen ausgezeichneten Überblick über Auflagen und Vertonungen von Metastasios Libretto L'eroe cinese bieten der Wikipedia-Eintrag „L'eroe cinese“, de.wikipedia.org/wiki/L\%E2\%80 \%999eroe_cinese, 15. Dezember 2019 sowie die einschlägigen Artikel: Don Neville: Art. Metastasio, Pietro (Antonio Domenico Bonaventura), in: Grove Music Online, online veröffentlicht 2001, doi.org/10.1093/gmo/9781561592630.article.53181, 16. Dezember 2019, und zu Metastasio allgemein vgl. Silke Leopold: Art. Metastasio, Pietro Antonio Domenico Bonaventura, in: Laurenz Lütteken (Hg.): MGG Online, Kassel u. a. 2016ff., zuerst veröffentlicht 2004, online veröffentlicht 2016, www.mgg-online.com/mgg/stable/14236, 15. Dezember 2019. 
auf die der Titel des Librettos hinweist, blieb im Kulturvergleich allerdings weitgehend unberücksichtigt. ${ }^{3}$

Im deutschen Sprachraum uraufgeführt und bald auch ins Deutsche übersetzt, bietet sich Der chinesische Held für einen interkulturellen deutsch-chinesischen Vergleich der Heldenkonzeption geradezu idealtypisch an: Er spielt nicht nur im alten China und behandelt einen in China populären Stoff, wie Metastasio im Vorwort andeutet, sondern hat zudem auch ein chinesisches Drama zur Vorlage.

\section{Der chinesische Prätext}

\subsection{Entstehung}

Die chinesische Quelle, auf die sich Metastasio bezieht, ist das erste chinesische Drama überhaupt, das in Europa übersetzt und bekannt wurde; überdies wurde der Stoff im 18. Jahrhundert mehrfach dramatisiert und adaptiert. Dem chinesischen Drama aus dem 13. Jahrhundert liegt als historische Quelle das einflussreiche Geschichtswerk Shiji des Historikers Sima Qian (145-90 v. Chr.) zugrunde. ${ }^{4}$ Darin findet sich die in das Jahr 583 v. Chr. datierte Geschichte von dem Waisenkind Zhao. Die Episode von der Rache eines vertauschten Kindes, das ein herrschertreuer Vater rettet, indem er seinen eigenen Sohn opfert, wurde in der YuanDynastie dramatisiert. Die Waise von Zhao übt große Rache (Zhaoshi gu'er da baochu) ist ein sogenanntes zaju, eine vieraktige Oper, Verfasser ist Ji Junxiang. Vollständig ediert wurde es erst im 16. Jahrhundert. ${ }^{5}$ Um es dem Zeitgeschmack der Ming-

3 Metastasios Libretto ist vergleichsweise wenig behandelt; vgl. vor allem Willy Richard Berger: China-Bild und China-Mode im Europa der Aufklärung, Köln/Wien 1990, bes. S. 198-201, Adrian Hsia: The Transplanted Chinese Orphan in England, France, Germany, Italy And his Repatriation to Hong Kong, in: ders.: Chinesia. The European Construction of China in the Literature of the $17^{\text {th }}$ and $18^{\text {th }}$ Centuries, Tübingen 1998, S. 75-98 sowie Chen Shouyi: The Chinese Orphan: A Yuan Play. Its Influence on European Drama of the Eighteenth Century, in: Adrian Hsia (Hg.): The Vision of China in the English Literature of the Seventeenth and Eighteenth Centuries, Hong Kong 1998, S.359-382, und Adrienne Ward: Pagodas in Play: China on the Eighteenth-Century Italian Opera Stage, Lewisburg 2010, bes. S. 98-111.

4 Die Episode von der Waise aus dem Hause Zhou ist allerdings nur bei Sima Qian überliefert.

5 Vgl. die chinesische Ausgabe: Zhaoshi gu'er, in: Yuanqu xuan, hg. von Zang Mouxun, Hangzhou 1998, S. 664-674; moderne Übersetzungen: Six Yuan Plays, übers. von Jung-en Liu, Harmondsworth, S. 21-25, S. 41-81. The Revenge of the Orphan of Chao, übers. von Pi-twan H. Wang, in: Renditions 9, 1978, S. 103-131 [engl.] und The Orphan of Zhao, in: The Orphan of Zhao and other Yuan Plays, hg. von Stephen H. West und Wilt L. Idema, New York und Chichester 2015, S. 49-56 [Einleitung] und S. 73-111 [Text]; nach dieser englischen Übersetzung wird im Folgenden zitiert.

Vgl. Wolfgang Bauer / Wolfgang Kubin: Ji Junxiang - Zhaoshi gu'er, in: Kindlers Literatur Lexikon in 18 Bänden, ${ }^{3} 2009$, hier Bd. 8, S. 363, sowie Wolfgang Kubin: Ji Junxiang (?-?): Die Waise von Zhao, in: Das traditionelle chinesische Theater: Vom Mongolendrama bis zur Pekinger Oper (Geschichte der chinesischen Literatur, Bd. 6), München 2009, S. 93-101 
Dynastie anzupassen, hat es der Herausgeber Zang Maoxum wohl verändert und um einen fünften Akt erweitert. So ist in der mutmaßlichen Originalversion der Yuan-Ära das Selbstopfer wichtiger als die Rache. Sie wird von dem Prinzen, der seine wahre Identität erfahren hat, am Ende des vierten Akts lediglich angekündigt. Dagegen wird die Rache im fünften Akt der Ming-Redaktion auf der Bühne blutig vollzogen. ${ }^{6}$

Das Drama hebt vorrangig auf die Rolle des Retters, Cheng Ying, und seine Beweggründe $\mathrm{ab}$, für den Fortbestand des Zhao-Clans sich und seinen eigenen Sohn zu opfern. Typisch für diese Frühform der chinesischen Oper ist auch, dass die Einheiten von Ort, Zeit und Handlung nicht beachtet werden und die Personen sich bei ihren Auftritten jeweils selbst vorstellen und ihre Absichten bekunden. Da komische, sentimentale und tragische Ereignisse miteinander abwechseln, lässt sich kaum entscheiden, ob es sich um eine Komödie (glückliches Ende) oder Tragödie (grausame Handlungen, unglückliches Ende) handelt.

\subsection{Handlung}

Das Stück spielt zur Zeit des Herrschers Jin Ling im 6. Jahrhundert v. Chr. Im Vorspiel berichtet der Bösewicht, General Tu'an Gu, von seiner Intrige gegen seinen Rivalen am Hof, den höchsten Zivilbeamten Zhao Dun. Diesem gelang zwar die Flucht, doch Tu'an Gu ließ alle dreihundert Mitglieder des Clans der Zhao töten. Bevor sich der Schwiegersohn von Zhao Dun erdolcht, mahnt er seine schwangere Frau, ihr gemeinsames Kind solle Rache für das Unrecht üben. ${ }^{7}$

Als (I. Akt) der Intrigant Tu'an Gu erfährt, dass die Tochter seines Rivalen Zhao Dun einen Sohn zur Welt gebracht hat, ordnet er dessen Ermordung an. Doch der dem Clan treu ergebene Arzt Cheng Ying entschließt sich zur Rettung des Neugeborenen, während die verzweifelte Prinzessin sich erhängt. Als Chen Ying das Kind, genannt die ,Waise aus dem Hause Zhao', in seinem Arztkoffer aus dem Palast schmuggelt, lässt ihn der diensthabende General aus Mitleid passieren, erdolcht sich aber anschließend, um einer Bestrafung zuvorzukommen.

Als daraufhin (II. Akt) der Bösewicht plant, alle Neugeborenen im Land zu töten, übergibt der Arzt Cheng Ying seinen eigenen kleinen Sohn einem alten, ehemaligen Offizier, der sich desillusioniert vom Hof aufs Land zurückgezogen hat, und verwahrt den neugeborenen Prinzen als eigenen Sohn bei sich zuhause.

sowie S. 106-107, sowie ders.: Ji Junxiang, in: Marc Herrmann u. a. (Hg.): Biographisches Handbuch chinesischer Schriftsteller. Leben und Werke, Berlin/New York 2011, S. 106107.

6 Allerdings erfolgt die Rache erst, nachdem sie von einem Abgesandten des Herrscherhauses legitimiert wurde.

7 „Wait until that child of mine has grown to manhood / And say, „For the three hundred of us - / Revenge! Take revenge for our injustice (The Orphan of Zhao [Anm. 5], Prolog [wedge], S. 76). 
Im dritten Akt gesteht der Arzt zum Schein, er habe die,Waise von Zhao' dem Offizier übergeben und verrate dies Geheimnis nur, um seinen eigenen neugeborenen Sohn zu retten. Als Tu'an Gu's Schergen bei dem alten Offizier in einem Versteck die angebliche, Waise aus dem Hause Zhao' finden, in Wahrheit der Arztsohn, wird dieser von Tu'an Gu getötet. Während Cheng Yin, der leibliche Vater, der Ermordung seines leiblichen Sohnes stoisch beiwohnen muss, nimmt sich der alte Offizier das Leben. Im Glauben, das Haus Zhao nun ganz vernichtet zu haben, verspricht Tu'an Gu dem Arzt, dessen neugeborenen Sohn zu adoptieren, nicht ahnend, dass dieser das vermeintlich getötete adlige Waisenkind ist.

Der vierte Akt setzt nach einem Zeitsprung von 20 Jahren ein, als Tu'an Gu die Herrschaft des Landes Jin an sich reißen will, um es an seinen Adoptivsohn, der den Namen Chen Bo führt, abzugeben. Darauf klärt der Arzt die Waise von Zhao über die Vorgeschichte auf, indem er dem jungen Mann eine Bilderrolle überreicht, in welcher die tragischen Episoden am Hof bildlich festgehalten sind. Dem Prinzen wird schlagartig klar, dass er die Waise von Zhao und sein Adoptivvater in Wahrheit der Mörder seiner leiblichen Eltern und seines Hauses ist. Die anaphorisch intensivierte Anagnorisis führt bei Cheng Bo zu einem hyperbolischen Racheschwur: ${ }^{8}$ Nachdem er in einer langen Arie seine Vergeltung drastisch ausmalt, versichert der Arzt in einem lakonischen Schlusswort die ,Waise' seiner Hilfe.

Der wohl später hinzugefügte fünfte Akt schildert die Exekution der Rache. Während Tu'an Gu auf grausame Art hingerichtet wird, darf die Waise nun wieder als Zhao Wu den Namen seines Hauses führen und wird in die alten Rechte eingesetzt. Zugleich wird sein Retter, der Arzt Cheng Ying, belohnt, wie auch diejenigen postum geehrt werden, die sich geopfert hatten, um das Leben des Waisenkindes zu retten. Dies bekräftigt die Schlussarie der Waisen Zhao Wu: „Die loyalen und gerechten Helden werden alle gerühmt und belohnt werden".9

Die französische Übersetzung des jesuitischen Gelehrten und China-Missionars Joseph Henri Marie de Prémare (1666-1736) erschien als erste europäische Version eines chinesischen Dramas überhaupt im Jahre 1735 in Jean-Baptiste Du Haldes (1674-1743) monumentaler, vierbändiger Description de la Chine et de la Tartarie chinoise (Beschreibung Chinas und der chinesischen Tartarei) (1735). Prémare überging allerdings die Arien in seiner Übersetzung und passte damit das Werk eher dem Stil einer europäischen Tragödie an. Doch wurde durch seine französische Version, die kurz darauf ins Englische und Deutsche übersetzt wurde, die „Chinesische

8 „He, he, he had our whole clan executed, / I, I, I will return by butchering nine generations of his" (The Orphan of Zhao [Anm. 5], IV. Akt, S. 106).

9 „Heroes loyal and righteous are all praised and rewarded, / Those who were military officers are restored to their commands, / Those who are destitute commoners are given sustenance, / Those who had died already are awarded a noble burial, / Those who are still alive receive titles and gifts. [...] In the history books we will leave our name, / For later generations to praise!" (The Orphan of Zhao [Anm. 5], V. Akt, Schlussarie, S. 111). 
Tragödie" Le petit Orphelin de La Maison de Tchao (Der junge Wayse aus dem Hause Tchao) europaweit bekannt und mehrfach dramatisch bearbeitet. ${ }^{10}$

\section{Intertextueller Vergleich}

Pietro Metastasios Anleihen bei dem Yuan-zaju sind offensichtlich, für das Helden-Konzept seiner Version sind aber die Differenzen aussagekräftiger. Sie zeigen sich in Struktur und Handlung, sowie einer anderen personellen Konstellation und thematischen Verschiebung.

\subsection{Strukturelle Differenzen}

Augenfällig ist die unterschiedliche Struktur der Stücke. Metastasio ändert Ort, Zeit und Handlung nach den klassizistischen Kriterien: Er vermeidet die Ortswechsel der chinesischen Vorlage. Einziger Schauplatz des Geschehens ist nurmehr die kaiserliche Residenz, und die zwanzig Jahre umfassende Handlungszeit reduziert er auf einen einzigen Tag. Die Heldentat, die Vertauschung seines neugeborenen Sohns mit dem Erbprinzen sowie die stoische Haltung, mit welcher der Arzt Cheng Ying die Ermordung des eigenen Kindes scheinbar fühllos erträgt, stehen im chinesischen zaju im Zentrum. Diesen dramatischen Höhepunkt der Vorlage spart Metastasio aus und distanziert ihn zur episch nachgetragenen Vorgeschichte. $\mathrm{Zu}$ dieser klassizistischen Regulierung passt auch die Verkürzung des fünfaktigen Prätexts auf drei Akte, wie sie für ein dramma per musica typisch ist. Auch endet das Libretto nicht mit einer blutigen Rache, sondern mit einer Rührszene.

\subsection{Geänderte Figurenkonstellation}

Der strukturellen Verdichtung entspricht eine neue Konstellation. Metastasio nobilitiert - passend zum Milieu des Kaiserhofs - das Personal und reduziert die Zahl der Dramenpersonen von zwölf auf fünf. Auch die Namen hat Metastasio geändert: So heißt der ,chinesische Held' bei ihm Leango, der Erbprinz Siveno alias Svenvango. Vor allem aber ist, und das ist die gravierendste Änderung, der böse

10 Tchao chi cou ell ou Le petit Orphelin de la Maison de Tchao. Tragédie chinoise trad. par Joseph Henri Marie de Prémare SJ, in: J[ean]-B[aptiste] Du Halde SJ: Description [...] de l'empire de la Chine et de la Tartarie Chinoise, 4 tomes, Paris 1735, t. III, S. 340-378; die deutsche Übersetzung: Tchao Chi cou ell. Oder Der junge Wayse aus dem Hause Tchao. Eine Chinesische Tragödie, in: Johann Baptista du Halde: Ausführliche Beschreibung des Chinesischen Reichs und der großen Tartarey, Dritter Theil, Rostock 1749, S. 418-444.

Die übersetzerische und produktive europäische Rezeption vermitteln überblickshaft Liu Wu-Chi: The Original Orphan of China, in: Comparative Literature 5, 1953, S. 193-212, Adrian Hsia: The Transplanted Chinese Orphan in England, France, Germany, Italy (Anm. 3), und Sherry J. Mou: A Child for all Ages: „The Orphan of Zhao“, in: Education About Asia 14, 2009, S. 23-28. 


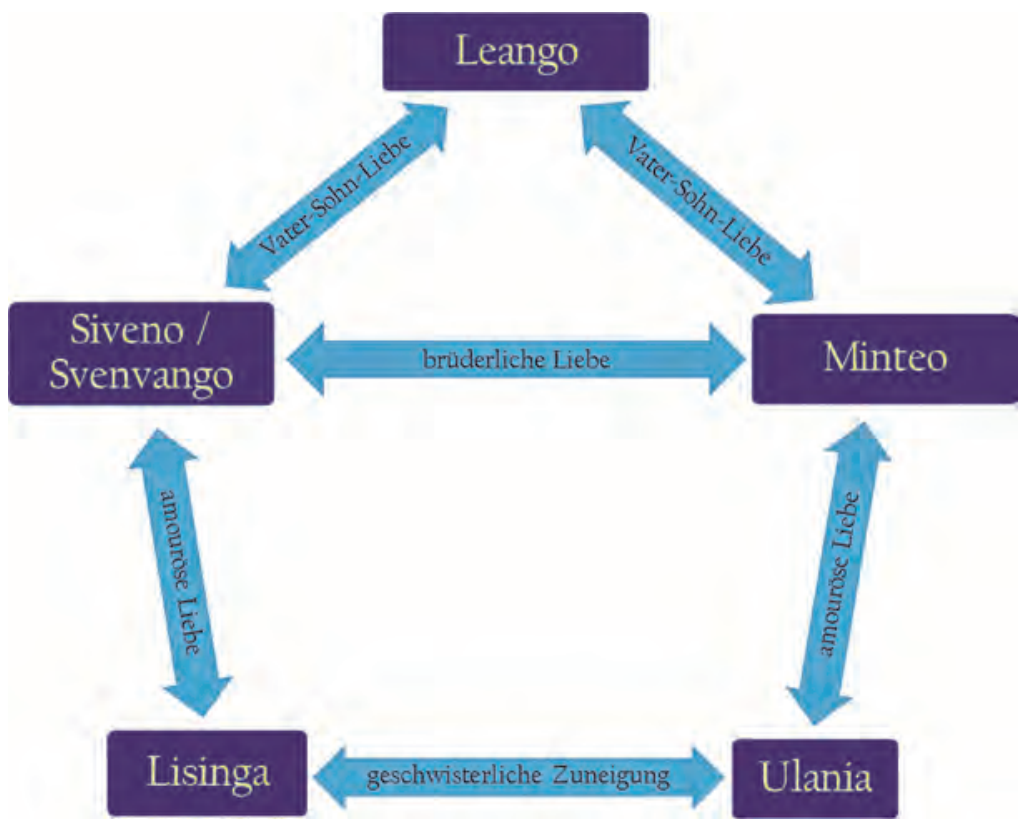

Abb. 1: Diagramm zur Figurenkonstellation.

Gegenspieler des Chinesischen Helden, Tu'an Gu, der im chinesischen zaju die Hauptrolle spielt, ganz gestrichen. ${ }^{11}$ Die andere wichtige Änderung der Konfiguration betrifft die neu hinzugefügte Figur des Minteo, des brüderlichen Freundes Sivenos und zugleich totgeglaubten Sohns Leangos. Er hat als Neugeborenes das Massaker schwer verletzt überlebt und ist von einem kaisertreuen Beamten großgezogen worden, im Glauben, er wäre der Erbprinz. Außerdem flankiert Metastasio die Handlung mit einem politisch grundierten Liebesgeschehen, indem er die zwei tartarischen Prinzessinnen Lisinga und deren Schwester Ulania einführt, die als Geiseln am Kaiserhof leben. Die Liebeshandlung intensiviert die Korrespondenzrelation der beiden Paare, die Leango spiegelsymmetrisch zugeordnet sind: Die dynamischen Interaktionsstrukturen, die sogenannten Figurenkonstellationen, ${ }^{12}$ lassen sich in einem Diagramm illustrieren (Abb. 1).

11 Metastasio folgt mit dieser Änderung mehr dem faktualen Gehalt als der fiktionalen Gestaltung der Episode, denn die chinesische Kaisergeschichte kennt nach Du Halde keinen Ministerstreit, sondern schreibt den Sturz des Kaisers Livango einem Volksaufstand zu.

12 Zur Terminologie ,Figurenkonstellation“ und ,Konfiguration“ vgl. Manfred Pfister: Das Drama, München 112001, bes. S. 232-235. 
Leango will nach zwanzigjähriger Regentschaft den von ihm geretteten Erbprinzen und an Sohnes statt großgezogenen Siveno als Herrscher inthronisieren und nutzt dessen Liebe zur tartarischen Prinzessin Lisinga zum Friedensabkommen mit der Tartarei. Zum Konflikt kommt es aber, weil Minteo, Sivenos Herzbruder und Leangos wahrer Sohn, sich für den rechtmäßigen Thronfolger hält. Zudem achtet Minteo Leango, ohne zu wissen, dass er dessen leiblicher Sohn ist, als Förderer und ,Vater. Da Minteo und Ulania, die andere tartarische Prinzessin, sich lieben, entsteht eine spiegelsymmetrische personale Korrespondenz zu Siveno und Lisinga. Damit verlagert Metastasio die tödliche Konkurrenz der Minister aus dem Yuan-zaju auf die nachfolgende Generation der Söhne, den Ziehsohn Siveno und den leiblichen Sohn Minteo.

Mit der neuen Konstellation nimmt Metastasio auch eine thematische Verschiebung vor. Die Konzentration auf nurmehr fünf Personen ohne einen wirklichen Gegenspieler und die Distanzierung des dramatischen Gewaltakts zur nachgetragenen Vorgeschichte verlagert den Konflikt in das Innere der Personen. Diese Internalisierung zeigt sich in der neuen zentralen Liebesthematik. Alle fünf Personen sind durch wechselseitige Liebesbeziehungen unterschiedlicher Art fest miteinander verbunden: Das Relationengeflecht umfasst neben der Vater-Sohn-Liebe (LeangoSiveno und Leango-Minteo) die freundschaftliche Liebe zwischen Siveno und Minteo bis zur geschwisterlichen Liebe zwischen Lisinga und Ulania.

Alle Liebesbeziehungen werden durch charakteristische Kippfiguren auf eine mehr oder minder starke Probe gestellt. In diesen Dramensituationen variiert und repetiert Metastasio die klassischen tragischen Elemente der Peripetie (,Glückswech$\mathrm{sel}^{\natural}$ ) und Anagnorisis (,Wiedererkennen'), die plötzliche Erkenntnis nach Irrtum: Das betrifft Leango, wenn er erfährt, dass Minteo, der geliebte Freund seines Ziehsohns Siveno, sein leiblicher Sohn ist; das betrifft Siveno, wenn er erfährt, dass Leango gar nicht sein Vater ist; noch stärker wechseln für Lisinga Glück und Unglück: Sie sieht sich zu einer politischen Zwangsheirat genötigt, bevor sie den geliebten Siveno für den ihr zugedachten Mann hält, doch daran zweifeln muss, bevor sie wieder daran glauben kann, anschließend jedoch Siveno tot wähnt, bis er glücklich von Minteo gerettet wird. Auf solche Wechselbäder der Gefühle hebt Metastasios Libretto ab. Die klassische Peripetie und Anagnorisis werden repetitiv eingesetzt und so zu einem desillusionierenden Dauermechanismus, in dem die Seelenstärke der Dramenpersonen erprobt und bewiesen wird.

\subsection{Internalisierung des Konflikts}

Metastasios Verschiebung des äußerlichen dramatischen Konflikts zum inneren seelischen Widerspruch zeigt sich lexikalisch-semantisch daran, dass Worte wie ,Seele' und ,Herz' [,alma', und ,cuore'] sowie ,Liebe' und ,Schmerz' [,amore' und ,dolore'] die Dialoge und Arien bestimmen. Die Kippfiguren, die Metastasios ,Drama für Musik` prägen, stellen jeweils die Identität der Personen in Frage. 
Daher reflektieren die Personen in Monologen und in a parte-Passagen immer wieder die Peripetien und Scheinwahrheiten. Vor allem Siveno erfährt gleich mehrfach solche Identitätskrisen:

Siveno: Giusto ciel, che mi avvene!

Son Svenvango o Siven!

Dove son! Chi son io! M'inganna il Padre!

Mi tradisce l'amico! ${ }^{13}$

SIVENUS: Gerechter Himmel, was wiederfähret mir! Bin ich Svenvangus, oder Sivenus? wo befinde ich mich? wer bin ich? betrüget mich der Vater? Verräth mich der Freund? ${ }^{14}$

Derartige Zweifel, die in der deutschen Version durch die Fragezeichen anstelle der Ausrufezeichen noch stärker akzentuiert sind, sind typisch für das Libretto. Immer wieder werden die Personen mit Situationen konfrontiert, die ihre Identität und Zukunft erschüttern und eine biographische Neuorientierung verlangen.

\section{Die Heldenkonzepte im Vergleich}

Mit Hilfe der intertextuellen Differenzen zwischen dem zaju der Yuan-Ära und dem aufklärerischen Libretto Metastasios lassen sich die unterschiedlichen Heldenkonzeptionen konturieren, die beiden Stücken zugrunde liegen.

\subsection{Die Waise von Zhao übt große Rache (Zhaoshi gu'er da baochu)}

Das chinesische zaju präsentiert die Heldentat eines dem Herrscherhaus loyalen Arztes, der aus wertrationalen oder traditionalen Motiven seinen eigenen Sohn opfert, um den Erbprinzen am Leben zu erhalten. ${ }^{15}$ Das Handeln des Arztes ist nicht situativ, sondern geplant und verlangt Ausdauer sowie Selbstverleugnung und Verstellung. So muss der Arzt mitansehen, wie sein leiblicher Sohn als vermeintlicher Erbprinz massakriert wird. Überdies stellt die heroische Tat keine rein individuelle Leistung, sondern ein Gemeinschaftswerk dar, an dem mehrere Helfer mitwirken. So der wachhabende General, der Cheng Ying mit dem Neugeborenen im Arztkoffer passieren lässt, und sich danach das Leben nimmt, und der alte Offizier, der den Sohn des Arztes als angeblichen Erbprinzen verwahrt und sich nach dessen Ermordung ebenfalls selbst tötet.

Inwieweit die Heldentat auch noch die Rache an dem Bösewicht einschließt, ist dagegen zweifelhaft. Denn zu den ausgreifenden und drastischen Plänen, in wel-

13 Metastasio: L'eroe cinese (Anm. 1), S. 33 [II 8].

14 Metastasio: Der Chinesische Held [L'eroe cinese, dt.], übers. von Johann Anton Koch, Bd. 6, Frankfurt am Main/Leipzig 1774, S. 89-164, hier S. 138 [II 8].

15 Vgl. Max Weber: Gesamtausgabe, Abt. 1., Bd. 23. Wirtschaft und Gesellschaft. Soziologie. Unvollendet 1919-1920, hg. von Knut Borchardt u. a., Tübingen 2013, S. 175-177, der das ,soziale Handeln' vierfach differenziert: Er unterscheidet (1) ,zweckrationales', (2) ,wertrationales', (3) ,affektuelles' und (4) ,traditionales' Handeln. 
chen der Erbprinz die grausame Tortur des Verräters ausmalt, bekennt Cheng Ying im Schlusswort lediglich seine Gefolgschaft. ${ }^{16}$ Allerdings ist die Rache bereits ein Motiv der heroischen Rettungs- und Tauschaktion, ${ }^{17}$ und als Cheng Ying den ehemaligen Offizier überredet, den vermeintlichen Prinz bei sich aufzunehmen, deutet er als symbolischen Lohn schon die Rache des Waisen von Zhao voraus: „He may take revenge for his father and mother. Wouldn't that be the best?" [,Er wird Rache nehmen für seinen Vater und seine Mutter. Wäre das nicht das beste?'] ${ }^{18}$ Nicht zufällig trägt das Stück den Titel Die Waise von Zhao übt große Rache (Zhaoshi gu'er da baochu) und hebt damit weniger auf die Rettungsaktion des Arztes als vielmehr auf die Legitimation der Rache ab, die das erwachsene Waisenkind im Schlussakt vollzieht.

Ein weiteres Motiv für das heroische Handeln ist die Hoffnung auf zeitüberdauernden Ruhm, wie es Cheng Ying eben dem retirierten Offizier verspricht: „If you make it possible for the Orphan of Zhao to survive, your name will be recorded in the history books and you will leave a reputation for all eternity" [,Wenn Du es der Waise von Zhao ermöglichst zu überleben, wird Dein Name in den Geschichtsbüchern erinnert werden und Du wirst Ruhm haben für alle Ewigkeit']. ${ }^{19}$ Eine Heroisierung, öffentlicher Ruhm, wird am Ende Cheng Ying und seinen Helfern zuteil, in der Schlussansprache der wieder zur Macht gelangten Waise von Zhao. Zugleich wird die Ehrung der Toten und Restitution der Geächteten zur militärischen Verpflichtung gegen äußere Feinde umgemünzt, um nach dem heroischen Vorbild das eigene Leben für das nationale Gemeinwohl zu riskieren:

Heroes loyal and righteous are all praised and rewarded.

Those who were military officers are restored to their commands,

Those who are destitute commoners are given sustenance,

Those who had died are awarded a noble burial.

Those who are still alive receive titles and gifts.

This grace is as broad as heaven!

And who would dare to make polite refusals?

We swear we will sacrifice our lives on the battlefield,

To make neighboring states all give us their allegiance.

In the history books we will leave our names,

For later generations to praise! ${ }^{20}$

16 „(Cheng Ying speaks:) Tomorrow the young master definitively will arrest that old traitor. I will have to follow him to be of assistance. (Exits.)" (The Orphan of Zhao [Anm. 5], S. 107 [Schluss 4. Akt]).

17 Auf die Rettung des Neugeborenen wird der Arzt schon von dessen Mutter im Hinblick auf spätere Rache eingeschworen: „Cheng Ying, [...] You must think of a way to hide this baby away so that later, when he's man, he can pay back the enemy of the Zhaos" (The Orphan of Zhao [Anm. 5], S. 107 [1. Akt]).

18 The Orphan of Zhao (Anm. 5), S. 89 [2. Akt].

19 The Orphan of Zhao (Anm. 5), S. 90 [2. Akt].

20 The Orphan of Zhao (Anm. 5), S. 111 [Schluss 5. Akt]. 
Damit wird die Heldentat Cheng Yings, die durch ihre Helfer bereits vergemeinschaftet war, nicht nur dem kollektiven Gedächtnis verpflichtend anvertraut, sondern auch zu einem verpflichtenden Präfigurat, einem Appell zur imitatio beroica an die Zeitgenossen und die Nachwelt, „for later generations“, denen die Namen und Taten in den Geschichtsbüchern als Vorbild vorgehalten werden.

\subsection{Der chinesische Held / L'eroe cinese}

Von der gewaltbereiten Heroisierung des chinesischen Prätexts unterscheidet sich Pietro Metastasios Bearbeitung des,Waisen von Zhao' fundamental. Da ein Gegenspieler fehlt, ist auch der Rachegedanke ausgeblendet. Es ist kein böses Individuum, sondern bezeichnenderweise das Volk, das den gefährdenden Faktor im Kampf um die Macht darstellt. Bei einem Volksaufstand hatte der Regent Leango den Erbprinzen gerettet, bereit, dafür seinen leiblichen Sohn zu opfern. Einem Volksaufstand fällt der Erbprinz am Ende des Stücks dann auch tatsächlich fast zum Opfer, bevor ihn sein ,Herzbruder ${ }^{6}$ Minteo aus höchster Not errettet.

Indem die eigentliche Heldentat, die Vertauschung der Neugeborenen und die Rettung des Erbprinzen, die im chinesischen zaju das Geschehen dominiert, zur epischen Vorgeschichte distanziert wird, verschiebt Metastasio das heroische Handeln auf die Inthronisation des neuen Kaisers. Die traditional motivierte Entscheidung des Regenten Leango, für seinen Ziehsohn, den Erbprinzen Siveno alias Svenvango, zurückzutreten, wird doppelt kompliziert: zum einen durch die Entscheidung des Thronrats, den verdienten Regenten Leango als Herrscher einzusetzen, zum andern durch die unvermutete Situation, dass der totgeglaubte Sohn Minteo doch noch am Leben ist und sich als Erbprinz wähnt, zumal ihn auch das Volk für den wahren Svenvango hält. Heroisch ist die Seelenstärke aller drei Beteiligten. Sie stellen allesamt in der Rührszene, die als offener Schluss das Singspiel beschließt, ihre Ambitionen zugunsten des Rivalen zurück.

SIVENO: Ah tu m'involi, amico (a Minteo.)

Il caro Padre mio.

MinTEO: Ma rendo al trono

Un Monarca sì degno. (Accennando Siveno.)

Siveno: Lascia ah lasciami il Padre, e prendi il regno. (Stringendo al petto la mano di Leango.)

LEANGO: Figli miei, cari figli, (Abbracciando or l'uno, or l'altro.)

Tacete per pietà. No ò vigore

Per sì teneri assalti. Astri clementi,

Disponete or di me. Rivenni il figlio:

Difesi il mio Sovrano:

Posso or morir, non ò vissuto in vano. ${ }^{21}$

21 Metastasio: L'eroe cinese (Anm. 1), S. 48 [Schlussszene]. 
SiVENUS: Ach du entziehst mir, Freund, (zum Minteus) meinen geliebten Vater.

MiNTEUS: Ich gebe aber dem Throne einen so würdigen Monarchen wieder. (Aufden Sivenus zeigend.)

Sivenus: Lasse, ach lasse mir den Vater, und nimm das Reich hin. (Die Hand des Leangus sich an die Brust drückend.)

LEANGUS: Meine Söhne, geliebte Söhne, (Bald den einen, bald den andern umarmend) schweiget aus Mitleiden. Ich habe nicht Kräfte genug für so zärtliche Anfälle. Gütige Gestirne schaltet nunmehr mit mir. Ich habe den Sohn wiedergefunden; ich habe nicht unnütz gelebet. ${ }^{22}$

Das Ende, das durch wechselseitige Überbietung an Seelengröße, einer heroischen magnanimitas, entsteht, lässt die Frage nach dem künftigen Herrscher offen, und auch Leango ist von der Situation affektuell sichtlich überfordert. Anders als im chinesischen Prätext wirkt angesichts des offenen Endes von Metastasios Libretto darum das Helden-Lob des Tutti-Finale (Coro) ganz unmotiviert: ${ }^{23}$
Sarà al mondo intero
Sarà chiara in ogni età,
Dell'Eroe di questo Impero
L'inudita fedeltà.

\author{
In aller Welt klar ausgebreit \\ Wird seyn, und wird auch jede Zeit \\ Die unerhörte Treue melden, \\ Von dieses Reiches Helden.
}

Bei Metastasio beruht das Heroische nicht mehr auf Überwindung eines feindlichen Gegenübers, sondern auf der altruistischen Überwindung der eigenen Person. Damit ähnelt der heroische Schluss des Chinesischen Helden einer Toleranzprobe, wie sie später Lessing in seiner Ringparabel in Nathan der Weise propagieren wird.

Bedenkt man, dass Alterität eine Variable darstellt, so bleibt zu erörtern, wie ,fremd', genauer, wie ,chinesisch' Metastasio die Heroik Leangos codiert und inszeniert. Exotisch sind nicht nur der Schauplatz, das Geschehen und die Figuren mit ihren fremden Namen, sondern auch deren Konstellation und heroisches Handeln. Chinesisch geprägt ist das setting, wie aus den minuziösen Szenenanweisungen hervorgeht. Nicht nur in der Architektur, sogar in der Natur kommt die kulturelle Differenz zum Tragen: „Die Thürme, die Dächer, die Pagoden, die Schiffe, ia selbst die Bäume, und alles was man siehet, zeiget den Unterschied, mit welchem sie von der Natur so wohl, als der Kunst, in einer so besondern Landschaft hervorgebracht werden" ${ }^{24}$ Auch zeitgenössische Illustrationen des Dramas betonen das ,Chinesische', wie etwa der Illustrator der französischen Ausgabe von 1780, der die abschließende Rührszene, in der Leango in Minteo seinen totgeglaubten Sohn erkennt, mit einer chinesischen Repoussoirfigur verfremdet (Abb. 2).

22 Metastasio: Der Chinesische Held (Anm. 14), S. 163-164.

23 Metastasio: L'eroe cinese (Anm. 1), S. 48 / Metastasio: Der Chinesische Held (Anm. 14), S. 164.

24 Metastasio: Der chinesische Held (Anm. 14), S. 115 [II 1]. Im italienischen Original lautet der Passus aus der Szenenanweisung zu II 1, der den Blick von der kaiserlichen Residenz in Singana auf den Fluss Vejo öffnet: „Le torri, i tetti, le pagodi, le navi, gli alberi istessi, e tutto ciò che si vede, ostenta la diversità, con la quale producono in clima così diverso non men la natura, che l'arte“ (Metastasio: L'eroe cinese [Anm. 1], S. 19). 


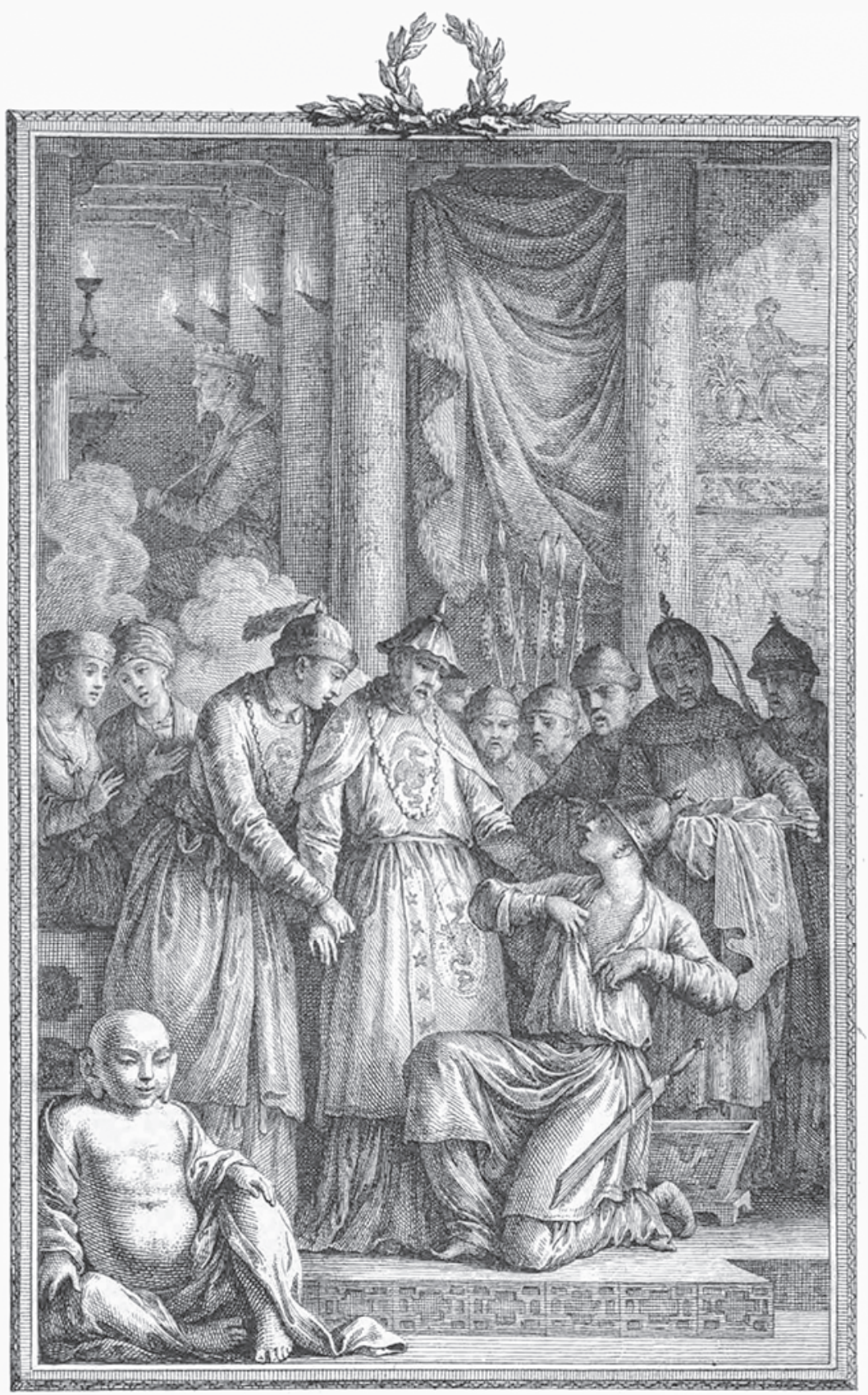

Abb. 2: Leango erkennt in Minteo seinen totgeglaubten Sohn. Kupferstich zur Schlussszene [III 9] von Pietro Metastasio, L'eroe cinese (in: Opere, Bd. 7, Paris 1780), mit dem Zitat von Minteos Rede: „Parlano queste cicatrici abbastanza. Osserva. Il caro mio genitor tu sei.“ 
Inszeniert wird aus europäischem Blick ein China als kulturelle Heterotopie, was religiösen Kultus, Architektur und hierarchisches Zeremoniell betrifft:

Innerer und beleuchteter Theil der grösseren kaiserlichen Pagode. Woselbst sowohl die Baukunst als die Auszierungen des prächtigen Gebäudes die Art und den Götzendienst der dasigen Völker ausdrücken.

Bonzen, Mandarinen des Krieges und der Gesetze, Grosse des Reichs, und Wache.

Bereits in der ersten Szenenanweisung wird das ,Chinesische“ vor allem mit Luxus und Verfeinerung korreliert, was in der Mitte des 18. Jahrhunderts das gängige Narrativ der damaligen China-Mode war: ${ }^{25}$

Appartementi nel Palazzo Imperiale destinati alle Tartare Prigioniere, distinti di strani pitture, di vasi trasparenti, di ricchi panni, di vivaci tapeti, e di tutto ciò che serve al Lusso, ed alla delizia Cinese.

Zimmer in dem kaiserlichen Pallast, bestimmt für die Tartarischen Gefangenen, geziert mit ausländischen Gemählden, mit durchsichtigen Gefäßen, mit reichen Decken, mit gemahlten Teppichen und mit allem demienigen, was zu dem Chinesischen Gepränge und zur Ergötzlichkeit dienet.

Der Hinweis auf ,Luxus` und „Gepränge“ spielt auf einen zivilisationskritischen Aspekt im China-Bild der Aufklärung an. Mit kultureller Überfeinerung hat etwa Rousseau die Unterwerfung der Chinesen unter die Mongolen erklärt. ${ }^{26}$ Tatsächlich zeichnet sich auch in Metastasios Libretto ein entsprechender Kulturkonflikt ab, wenn die beiden als Geiseln gefangenen tartarischen Prinzessinnen durch einen Diktatfrieden der Tartaren freikommen. Er schließt als Bedingung die Verheiratung Lisingas mit dem chinesischen Erbprinzen ein, und dank der militärischen Macht der Tartaren wird auch der chinesische Volksaufstand im Schlussakt niedergeschlagen. Diese Rahmenhandlung passt zu dem zeitgenössischen Erklärungsmuster von der luxurierenden und verfeinerten chinesischen Aristokratie. Mit der ehelichen Verbindung der Tartarenprinzessinnen mit den chinesischen Prinzen wird eine vitalisierende Blutauffrischung als Remedium gegen die Überfeinerung avisiert.

Versteht man den chinesischen Helden Leango als dynamisch konzipierte Figur, so spiegeln sich in ihm Stärke wie Schwäche der chinesischen Kultur, freilich aus europäischer Perspektive. Während Leango sich zwanzig Jahre zuvor noch mutig dem marodierenden Pöbel entgegenstellte und so das Leben des Erbprinzen rettete, erweist er sich in der abschließenden Rührszene, als er in Minteo den totgewähnten Sohn wiederfindet, gefühlsmäßig überfordert und handlungsunfä-

25 Metastasio: L'eroe cinese (Anm. 1), S. 5 / Metastasio: Der Chinesische Held (Anm. 14), S. 93.

26 Gegen Voltaire hatte Rosseau in seiner Preisschrift Si le rétablissement des sciences et des arts a contribué à épurer les moeurs (1750) am Beispiel des wissenschaftlich und künstlerisch hoch entwickelten China, das den „unwissenden und plumpen Tartaren“ zum Opfer gefallen sei, gezeigt, dass Wissenschaften und Künste zu Sittenverfall und militärischer Schwäche führten; vgl. Hsia: Chinesia (Anm. 3), S. 100. 
hig. Damit stellt Metastasios Libretto in Leango die beiden Seiten einer zivilisierten Hochkultur und eines vollkommenen Humanismus dar: Leango entspricht dem aufklärerischen Ideal eines kultivierten mitmenschlichen Umgangs, andererseits erweist er sich vor lauter Humanität als im Handeln fast paralysiert. Es entbehrt nicht der Ironie, dass die großmütige Heroik des Verzichts, die das Libretto mit seinem offenen Ende sowohl als Muster propagiert wie in ihrer Schwäche kritisiert, ausgerechnet ein Rachestück zum Prätext hat.

\section{Ausblick}

$\mathrm{Zu}$ wenig hat man bislang den Diskurscharakter von Metastasios Chinesischem Held beachtet. Das Stück kritisiert mit der projektiv überformten Darstellung chinesischer Heroik europäische Heldenkonzepte, die bis zur Mitte des 18. Jahrhundert militärisch und bellizistisch fundiert waren. Zugleich knüpft er an den zeitgenössischen China-Diskurs an, indem er in Leangos Handlungsunfähigkeit die Schwäche einer solch überfeinerten und verinnerten Heroik andeutet. Lohnend wäre es zu untersuchen, wie die europäische Dramatik des 18. Jahrhunderts auf die im Chinesischen Helden sowohl propagierte als auch kritisierte exotisch verbrämte interiorisierte Heroik reagierte. Kritisch revidiert Voltaire in seiner Tragödie L'Orphelin de la Chine (1753/55) Metastasios Verklärung. Voltaire greift zwar denselben Stoff auf, historisiert und aktualisiert ihn aber stärker, ohne den Rachegedanken auszublenden. Auch in England gibt es mit Arthur Murphys Drama The Orphan of China (1756) eine eher bellizistische Antwort auf Metastasios humanitäre Heroik. Die deutsche Rezeption dieses Stoffs ist bisher noch gar nicht vergleichend betrachtet worden. Neben den übersetzerischen Adaptationen von Metastasios Libretto und Voltaires Trauerspiel haben prominente Autoren der deutschen Klassik wie Wieland in Der goldene Spiegel oder die Könige von Scheschian (1772) und Goethe den Stoff aufgegriffen. Möglicherweise ist das Humanitätsideal der deutschen Klassik gar nicht nur von der Klassischen Antike geprägt, wie man bisher immer annahm. Vielleicht spielt die mitmenschliche Heroik des Chinesischen Helden, dessen Spuren in Goethes Elpenor-Fragment unverkennbar sind, eine größere Rolle, als man bisher weiß. Aber das bleibt vorerst nur eine Mutmaßung.

\section{Abbildungsnachweise}

Abb. 1: Achim Aurnhammer.

Abb. 2: Wikimedia Commons, Fotograf: Rodomonte, upload.wikimedia.org/ wikipedia/commons/0/06/Metastasio_-_L\%E2\%80\%99eroe_cinese_-Herissant_Vol.07_-_Paris_1780.png. 